\title{
Cranial nerve palsy in post partum female
}

\begin{abstract}
Pre-eclampsia is a condition which is related to pregnancy and has various clinical manifestations as well. It usually presents in pregnant females around twentieth week of pregnancy. The classic signs include high blood pressure and proteinuria. Some patients develop neurological signs as well which include sensory and motor involvement. We present a case of female presenting as isolated third nerve palsy one week post partum.
\end{abstract}

Keywords: third nerve palsy, preeclampsia, pregnancy, hypertension; proteinuria

\author{
Volume 6 Issue 6 - 2017
}

\author{
Waseem Qureshi, Freia McGregor, Jyotin \\ Pandit \\ Department of Ophthalmology, Great Western Hospital, UK
}

Correspondence: Waseem Qureshi, Department of Ophthalmology, Great Western Hospital, UK, Tel 0798642068I, Emailwazeem77@hotmail.com

Received: November 02, 2016 | Published: May I, 2017
Abbreviations: ANA, antinuclear antibody; ANCA, antineutrophil cytoplasmic; ACE, angiotensin converting enzyme; HIV, human immunodeficiency virus; CT, computerized tomography; MRI, magnetic resonance imaging; MRA, magnetic resonance angiography

\section{Introduction}

The pre-eclampsia presenting as focal neurological defect is rare and not documented in peer review journals. Our patient, a female, was in her thirties and less than a week post partum. She presented with isolated double vision and signs of third nerve palsy. At presentation, there was a complete pupil involving $3^{\text {rd }}$ nerve palsy affecting the left eye. There were no other associated neurological symptoms in history or elicited on examination. Initial investigations included the following tests: Full blood count, blood calcium levels, serum antinuclear antibody (ANA), anti-neutrophil cytoplasmic (ANCA), antiphospholipid antibody and angiotensin converting enzyme (ACE) within normal limits. Human Immunodeficiency virus (HIV), syphilis, lyme serology and neuroimaging including
Computerized Tomography, Magnetic Resonance Imaging and Angiography (CT, MRI, MRA) and lumbar puncture were within normal range. No infectious, compressive or autoimmune cause was found. Computerized tomography of thorax was performed due to palpable cervical lymph node but this did not reveal any abnormality. ${ }^{1}$

The patient remained relatively asymptomatic, pain free throughout and had complete lid occlusion due to her left ptotic eyelid (Figure 1). As the ptosis resolved she was provided with a patch. She was lost to follow up and had almost complete recovery till her last orthoptic assessment on follow up. Third nerve palsy can present in a variety of ways to eye casualty; varying from an acute aneurysm causing direct compression on the nerve to ischaemic changes and pre-existing copathology. The symptoms arise from weakness of the levator muscle resulting in ptosis, intact unopposed action of the lateral rectus with the eye in an abducted position and intorsion of the eye at rest due to intact superior oblique action which increases on attempted downgaze resulting in diplopia. Involvement of the parasympathetic fibres cause pupil dilation associated with defective accommodation. ${ }^{2}$

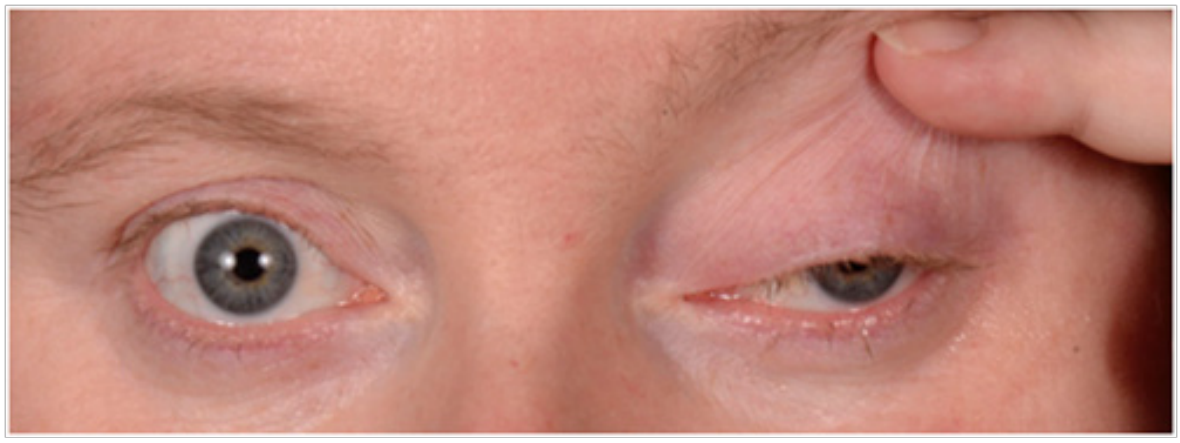

Figure I Left third nerve palsy, ptosis, and eyeball turned down and out.

The most common aetiology of pupil-sparing $3^{\text {rd }}$ nerve palsy is microvascular as in diabetes and hypertension. Spontaneous recovery is seen mostly within 3 months. It is often quoted 1,2 that the presence of pain is useful in differentiating aneurysmal and microvascular causes which has implications on urgent management, however diabetic $3^{\text {rd }}$ nerve palsy is often associated with periorbital pain. Aneurysm of the posterior communicating artery at its junction with the internal carotid is a very important cause of isolated $3^{\text {rd }}$ nerve palsy (usually painful) with pupil involvement due to the clinical implications of an enlarging aneurysm. Urgent neurosurgical intervention is required. Trauma, both direct and secondary to subdural haematoma with uncal herniation is also documented. Other rarer causes include vasculitis, migraine, myasethenia gravis and alterations in intracranial pressure. The most unique features are as follows. There are only a few published cases of $3^{\text {rd }}$ nerve palsy due to pre-eclampsia. ${ }^{3}$ The interesting feature in this case is that it occurred five weeks post-partum in a pregnancy with pre-eclampsia. There are two proposed mechanisms for nerve palsies in pre-eclampsia - 1) focal reactive vas0coconstriction and 
local hypoperfusion, 2) hyperperfusion and break-down of the bloodbrain barrier that occurs with rising hypertension causing extravasation into brain parenchyma. Due to large fluctuations in blood pressure noted by a midwifery team in the month post-delivery, it is likely that this case represents more of an ischaemic picture than vasospasm. Once life-threatening causes have been excluded, treatment is usually conservative, as in this case. Surgical intervention is usually reserved for cases where all spontaneous improvement has ceased and when it is over six months from the date of onset.

\section{Acknowledgements}

None.

\section{Conflicts of interest}

The authors declare there are no conflicts of interest.

\section{References}

1. Bonebrake RG, Fleming AD, Carignan EM, et al. Severe preeclampsia presenting as third nerve palsy. Am J Perinatol. 2004;21(3):153-155.

2. Chutatape A, Teoh WH. Third nerve palsy associated with preeclampsia and HELLP syndrome. J Anesth. 2013;27(5):757-760.

3. Watanabe H, Hamada H, Fujiki Y, et al. Third nerve palsy and serous retinal detachment with preeclampsia. Hypertens Pregnancy. 2006;25(1):33-35. 